\title{
HAEMANGIOENDOTHELIOMA OF THE LIVER SIMULATING CONGENITAL HEART DISEASE IN AN INFANT
}

\author{
BY \\ C. BLAXLAND LEVICK and J. RUBIE \\ From the Victoria Hospital for Children, London, and St. George's Hospital, London
}

(RECEIVED FOR PUBLICATION JULY 25, 1952)

The association in a young infant of persistent central cyanosis, increased by crying and feeding, with a systolic murmur heard all over the precordium, almost always indicates congenital heart disease. In the present case the existence of those features led to this diagnosis, yet the necropsy failed to reveal such a lesion. In fact the infant suffered from a haemangioendothelioma of the liver.

\section{Case Report}

M.H., aged 20 days, was admitted to hospital on account of dyspnoea and cyanosis. She had been normal at birth, apart from some blue asphyxia which quickly responded to treatment. Her birth weight was $7 \mathrm{lb} .13 \mathrm{oz}$.

Her mother said that the infant had always had difficulty in breathing, had seemed to breathe rapidly, and had tired easily during feeding. When 14 days old she had an attack lasting about 15 minutes, in which she became very blue and appeared to have difficulty in breathing. When 20 days old she had a similar attack which did not cease spontaneously. She was admitted to hospital about four hours after its onset.

On examination her nutrition was fairly good. She was too ill to weigh, but appeared to be average size for her age. There was moderate general cyanosis and the external jugular veins were slightly distended. The alae nasi were active. Her temperature was $99 \cdot 6^{\circ} \mathrm{F}$., pulse 180 (regular) and respirations 60. There were no congenital surface stigmata, no petechiae or naevi, and no clubbing of fingers or toes.

The left side of the thorax was slightly larger than the right; slight bilateral lower rib recession was seen. The cardiac apex beat was displaced to the left, just outside the mid-clavicular line. No thrill was felt in any area. The mitral sounds were spaced in even tapping rhythm. The pulmonary second sound was normal. A blowing systolic murmur could be heard all over the precordium, maximal at the middle of the lower end of the sternum. A short soft diastolic murmur was heard on both sides of the xiphisternum. The blood pressure was not taken. Femoral pulsation was normal. No subcutaneous oedema or serous effusion was observed.
Fine crepitations were present at both lung bases. The liver was enlarged by one fingerbreadth but there was no surface irregularity, pulsation or thrill. The spleen was not enlarged, and there was no undue abdominal distension.

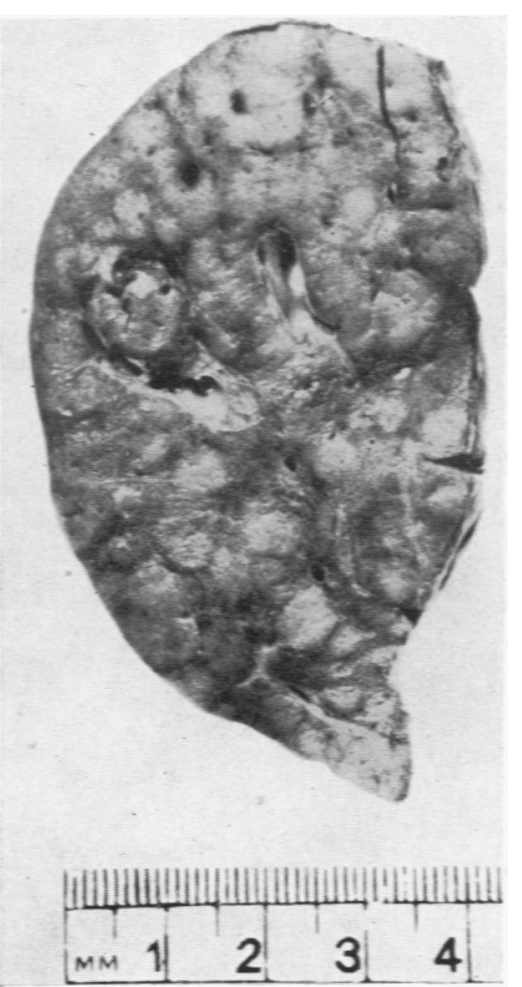

Fig. 1.-Cut surface of the liver showing angiomatous masses appearing as pale, ill-defined nodules.

The cyanosis was slowly, but only partially, relieved in an oxygen tent; the heart rate fell to 150 , and the respirations to 50 per minute. 
A radiograph of the chest, taken by a portable machine, showed gross cardiac enlargement, mainly to the right, but also to the left, and slight vascular congestion of the lung fields. The pulmonary arteries were not visualized.

A full series of electrocardiograms gave a regular sinus tachycardia of 150 per minute and right ventricular hypertrophy in a vertical heart with clockwise rotation.

The tendency to cyanosis appeared to increase rapidly until it was possible to remove the baby from the oxygen tent for only a few moments at a time. X-ray screening was not practicable. Cyanosis then became extreme, and the child died eight days after admission.

Extract from Necropsy Report. There was no external evidence of congenital abnormality. The heart was considerably enlarged; both ventricles were hypertrophied, especially the right. The interventricular septum was intact. The foramen ovale was patent but largely covered by a crescentic fold of endocardium, and was normal for the baby's age; nothing in its appearance suggested a venous-arterial shunt. The valves and great vessels were normal, and the ductus arteriosus admitted a fine probe with difficulty and was clearly closing normally.

The lungs were greatly congested. The endocrine system was normal. The kidneys and spleen showed slight congestion. The brain and meninges were normal.

The liver was moderately enlarged. Most of the normal parenchyma was replaced by numerous nodules, each measuring about $1 \mathrm{~cm}$. in diameter. These nodules were distributed throughout both lobes; some were visible on the surface and showed umbilication. No similar nodules were found elsewhere in the body. The gall bladder, extra-hepatic biliary tract and pancreas were normal. The alimentary system was otherwise normal.

Histology. There was widespread replacement of the

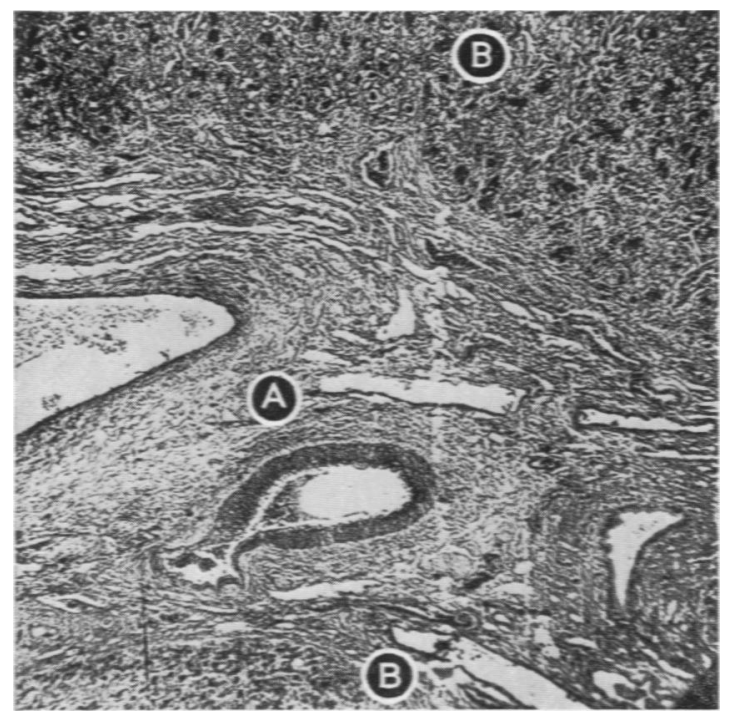

Fig. 2.-Section of liver (low power) showing portal tract (A) and liver lobules disorganized by proliferating vasoformative tissue (B). liver parenchyma by haemangioma. The nodules described above were composed of proliferating vasoformative cells and sinusoidal blood channels. The lungs, spleen and kidneys showed the features of venous congestion, but no other congenital abnormality was found.

In our case the diagnosis of congenital heart disease appeared to be fully justified before death. It seems likely that, as necropsy showed no such lesion, the heart failure was due to the arterio-venous shunt. It is interesting to note that in our case right-sided failure predominated, associated with cyanosis and venous engorgement. This has been noted in cases previously described, but usually other clinical features, such as cutaneous lesions, were present together with some of the clinical features described by Kunstadter (1933).

\section{Pathological Considerations}

Andries and Kaump (1944) classify haemangioma of the liver as (1) benign (large or small, single or multiple) or (2) malignant.

Stout (1943) has pointed out that theoretically malignant vascular tumours could arise from the endothelial cells, the perithelial cells, or the smooth muscle. The haemangioendothelioma is the commonest, the haemangiopericytoma has been described (Stout and Murray, 1942), but there is doubt about the leiomyosarcoma. A benign leiomyoma has been described (Stout, 1937, 1943).

Howard (1936) regards these lesions as multiple

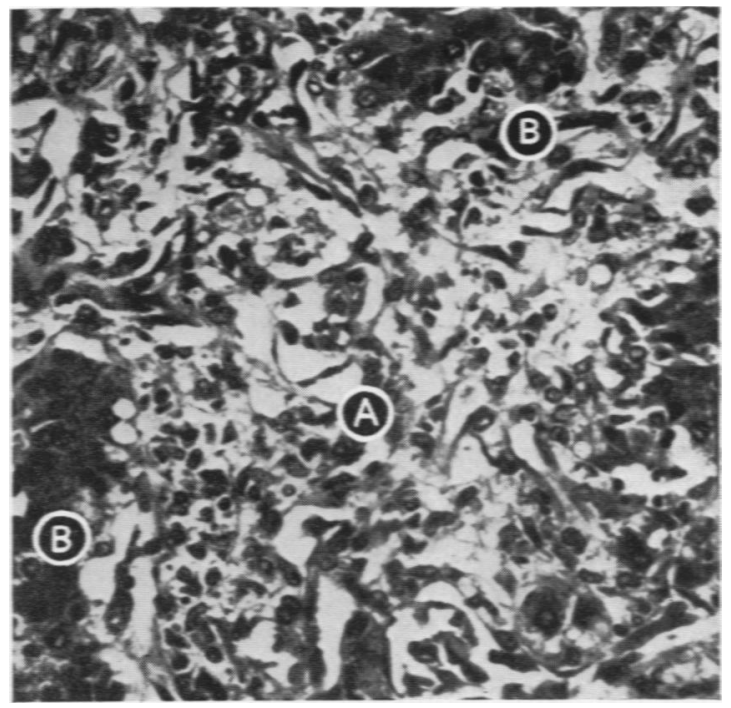

Fig. 3.- Section of liver (high power) showing vasoformative cells (A) separating the parenchyma into small, irregular groups of cells (B). 
congenital tissue defects rather than malignant lesions.

A true hamartoma or vascular malformation is not a neoplasm; it has no power of disproportionate growth but enlarges with the tissues, apart from incidents such as haemorrhage or sepsis.

Foote (1919) considers all these lesions to be congenital, arising from endothelium which has not lost its embryonic characteristics. When this occurs in adults the benign angioma has either reverted to embryonic type or resulted from foetal inclusions. He recognizes a separate group of malignant tumours which probably occur mainly in adults.

Thus it is seen that there is controversy concerning the designation of the scattered lesions. The two points of view are, on the one hand, a primary malignant lesion with metastases, and, on the other, multiple congenital lesions. Rabson (1938) considers both explanations possible. We believe that a congenital tissue defect is the more likely explanation in our own case and probably in others arising in early infancy. Nevertheless, histological examination showed a degree of cellularity which one would associate with local malignancy, and in Fig. 3 the cellular elements can be seen infiltrating the liver cells.

\section{Discussion}

The term haemangioendothelioma was introduced by Mallory (1908) to describe a malignant vascular lesion of the skin but it has since been extended to include lesions elsewhere. Haemangioendothelioma of the liver has been described with and without similar lesions in other organs. Similarly haemangioendothelioma of various organs, yet without liver involvement, has also been described (Wollstein, 1931).

Kunstadter (1933) described a case of haemangioendothelioma of the liver in a child of 5 months, who had been noted to have an enlarged abdomen and pallor from the age of 6 weeks, and developed dyspnoea and cyanosis at the age of 4 months when lying down. Vomiting and constipation followed, and the infant died after laparotomy. Kunstadter reviewed 14 previously reported cases in infancy.
Cases have since been described by various authors, including Taylor and Moore (1933), Howard (1936), Dordick (1938), Schumann (1941), Blauel (1942), Andries and Kaump (1944), Schwartz (1945), Berezin, Scharnoff and Stein (1948) and Sweed and Weinberg (1950).

From the cases described in the literature it seems that the most constant presenting feature was abdominal enlargement, often accompanied by some gastro-intestinal disturbance such as vomiting, constipation or stool abnormality. Anaemia was a frequent finding, and jaundice and ascites were noted in two cases. A palpable abdominal mass. usually under the right costal margin, was noted in other cases. The condition of the infants deteriorated, and they usually died of exhaustion and respiratory embarrassment, sometimes with cyanosis.

\section{Summary}

A case of haemangioendothelioma of the liver is described in the neonatal period. Dyspnoea, feeding difficulty, central cyanosis, a systolic murmur. and a grossly enlarged heart suggested that a congenital heart lesion with heart failure was present. At necropsy there was no such lesion and the diagnosis was established.

We wish to thank Dr. N. F. Gowing who performed the necropsy and reported on the sections, and Professor T. Crawford of the Pathology Department, St. George's Hospital, London.

\section{REFERENCES}

Andries, G. H. and Kaump, D. H. (1944). Amer. J. clin. Path., 14, 489.

Berezin, S. W., Sharnoff, J. G. and Stein, J. D. (1948). Je'n Engl. J. Med., 238, 906.

Blauel, I. (1942). Mschr. Kinderheilk., 91.345.

Dordick, J. R. (1938). Amer. J. Dis. Child., 55. $\$ \$ 9$.

Foote, J. (1919). J. Amer. med. Ass., 73, 1042.

Howard, W. A. (1936). J. Pediat., 8, 588.

Kunstadter, R. H. (1933). Amer. J. Dis. Child., 46, 803

Mallory, F. B. (1908). J. exp. Med., 10, 575.

Rabson, S. M. (1938). Arch. Path., Chicago, 25. 185

Schumann, H. D. (1941). Frankfurt Z. Path., 55. 1.

Schwartz, A. R. (1945). Arch. Pediat., 62, 1 .

Stout, A. P. (1937). Amer. J. Cancer, 29, 435.

- (1943). Ann. Surg., 118. 445

and Murray, M. R.' (1942). Ibid., 116, 26

Sweed, A. and Weinberg, T. (1950). Amer. J. Dis. Child., 80, 436

Taylor, A. C. and Moore, E. (1933). Amer. J. Cancer, 19, 31.

Wollstein, M. (1931). Arch. Path., Chicago, 12. 562. 